ganglion focusing the importance of the floor of the anterior epitympanum.

doi:10.1017/S0022215116006162

\section{ID: IP120}

Cholesteatoma in children, is it really particular?

\section{Presenting Author: Benzamit Makhlouf}

Benzamit Makhlouf $^{1}$, Djarmouni Nabila ${ }^{2}$, Benamira Mohamed $^{3}$, Kehal Youcef ${ }^{3}$

${ }^{1}$ Chawki \& Achwak Clinic, SETIF, ${ }^{2}$ Ent Department, Ferhat Abbas University, ${ }^{3}$ Chawki et Achwak Clinic

\section{Learning Objectives:}

Introduction: Cholesteatoma is a serious middle ear disease, affecting both adults and children. it is more special in children. Occurred on a pneumatised mastoid, cholesteatoma in children is more aggressive with a great potential of extension and a high tendency to recurrence. Although in literature many authors support this hypothesis, others still disagree with this point of view at the present time. Therefore, the particularity of cholesteatoma in children is a reality or just a myth? Our study aims to emphasize on this issue.

Materials and methods: with a longitudinal-type study on 82 cases of acquired cholesteatoma in children at ENT department of Ferhat Abbas university and Chawki \& Achwak clinic between January 2004 and December 2015. The aim of this work is to illustrate the clinical,para clinical and therapeutic features of cholestatoma in the pediatric population and highlight the main characteristics.

Results: The main reason for consultation is largely driven by the fetid otorrhea $(96.5 \%)$, hearing loss, however,is well behind $(66.7 \%)$. It is worth noting that Tubal dysfunction, adaptation disease, allergy are very common and haracterize children. CT scan is the imaging method of choice in the preoperative evaluation. It provides useful details, particularly regarding the pneumatisation of the mastoid.Thus, confirming that cholesteatoma in children occurs on a very pneumatised mastoid which usually belongs to younger children. Granulation tissue in the middle ear and the mastoid cavities, denuded facial nerve, very extensive cholesteatoma are the most common difficulties to remove the disease and to prevent the recurrence which is absolutely higher than that observed in adults.

Conclusion: Cholesteatoma of the child is special because the child himself is special. The large clinical latency And the misdiagnoses complicate not only the task of the surgeon but also the prognosis with a high potential of recurrence whatever the technique used.
doi:10.1017/S0022215116006174

\section{ID: IP121}

Endaural Approach through Post auricular Incision

Presenting Author: Bulent Mamikoglu Bulent Mamikoglu ${ }^{1}$, Bulent Mamikoglu ${ }^{2}$

${ }^{1}$ Chicago Dizziness and Hearing, ${ }^{2}$ Chicago Dizziness and Hearing, and U of Illinois at Peoria Medical School, Department of Neurosurgery

Learning Objectives: Endaural approach can be performed through postauricular incision. Author has 20 years of experience using endaural approach in cholesteatoma surgery. Author will discuss the surgical technique, patient selection, difference in surgical drilling compared to corticol mastoidectomy, and results. Endaural approach allows minimally invasive surgery for cholesteatoma patients with fast recovery and smaller mastoid cavity for post operative care. Hearing results are competitive to corticol mastoidectomy results. Case selection is important but also surgery can easily swith into canal down procedure if the pathology is larger than predicted before surgery. Cartilage grafting is important part of the surgery and grafts material is usually optained from auricular concha rather than tragus.

doi:10.1017/S0022215116006186

\section{ID: IP122}

Comparative study on different graft tissues for simple myringoplasty

\section{Presenting Author: Francesco Mancini}

Francesco Mancini, Tommaso Sorrentino, Nader Nassif, Luca Oscar Redaelli de Zinis

Spedali Civili di Brescia

Learning Objectives: Comparison of three different autologous graft tissues in simple myringoplasty in term of effectiveness and hearing gain.

Introduction: In case of chronic otitis medica it is possible to reconstruct the defect of the eardrum with different autologous materials: fascia $(\mathrm{F})$, perichondrium $(\mathrm{P})$, or cartilage C. Last tissue shows greater resistance but might reduce the gain of the auditory canal due to its thickness. Aim of the study is to compare the effectiveness and the success in auditive gain of the 3 different tissues in simple myringoplasty. 
Material and Methods: All simple myringoplasty of the last 3 years have been revaluated. Exclusion criteria were the presence of a cholesteatoma and chronic otitis. An otoscopic picture of both ears was taken for each patient. The site of perforation was classified into anterior, posterior and subtotal. A PTA, according to the guidelines of the AAOHNS has been performed before and 2 months after surgery.

Results: A total of 123 patients undergoing simple myringoplasty was identified. In 33 patients we used $\mathrm{C}$, in $33 \mathrm{~F}$ and in $26 \mathrm{P}$. The overall failure rate was $10 \%$, divided in: $12.1 \%$ for $\mathrm{C}$ (plus a further $12.1 \%$ of microperforation all repaired), $2.7 \%$ for F, $18.2 \%$ for P. The status of the contralateral ear showed it was pathological in $48.5 \%$ of cases of $\mathrm{C}$, $16.6 \% \mathrm{~F}$ and $18.2 \%$ of $\mathrm{P}$. The site of the perforation was anterior in $48.5 \%$ of $\mathrm{C}, 41.6 \%$ of $\mathrm{F}$ and $40.1 \%$ of $\mathrm{P}$; posterior in $12.1 \%$ of C, $13.8 \%$ of $\mathrm{F}$ and $45.4 \%$ of $\mathrm{P}$; subtotal in $39.4 \%$ of $\mathrm{C}, 44.4 \mathrm{~F}$ and $13.6 \%$ of $\mathrm{P}$. The ABG was $26.9 \mathrm{~dB}$ for the preoperative $\mathrm{C}, 20.7 \mathrm{~dB}$ for $\mathrm{F}$ and $18.6 \mathrm{~dB}$ for and $\mathrm{P}$; The postoperative $\mathrm{ABG}$ was $17.3 \mathrm{~dB}$ for $\mathrm{C}, 13.1 \mathrm{~dB}$ for $\mathrm{F}$ and $11.5 \mathrm{~dB}$ for $\mathrm{P}$. The auditive gain (difference of $\mathrm{ABG}$ pre and postop) was $9.5 \mathrm{~dB}$ for $\mathrm{C}, 7.5 \mathrm{~dB}$ for $\mathrm{F}$ and $7 \mathrm{~dB}$ for $\mathrm{P}$.

Conclusions: The results show an overall success rate in line with the literature. It emerges that $\mathrm{F}$ has the best success rate but $\mathrm{C}$ is used mostly in cases where the contralateral ear is pathological. The auditory gain is comparable, even if $\mathrm{C}$ is chosen in the cases with a worse initial ABG.

doi:10.1017/S0022215116006198

\section{ID: IP123}

\section{An evaluation of the NHS Clinical Commissioning Policy on Bone Anchored Hearing Aids}

Presenting Author: Rishi Mandavia

\author{
Rishi Mandavia ${ }^{1}$, Samit Unadkat ${ }^{2}$, Anne Schilder ${ }^{1}$ \\ ${ }^{1}$ Ear Institute, University College London, \\ ${ }^{2}$ London North Thames ENT Registrar
}

\section{Learning Objectives:}

Introduction: The NHS Commissioning Policy on Bone Anchored Hearing Aids (BAHA) identifies the criteria for the commissioning of BAHA services and therefore has a major impact on patient access to care. This paper aims to evaluate the evidence base informing the NHS Commissioning Policy on BAHAs. We also aim to produce recommendations on BAHA policy development.

Methods: This study was conducted in two parts.

1) Critical assessment of the evidence based informing the NHS Commissioning Policy on BAHAs. Quality of included articles and the overall strength of the policy were assessed using the Grading of Recommendations Assessment, Development, and Evaluation (GRADE) System.
2) Systematic review of the literature on BAHAs published since the release of NHS Commissioning Policy. Papers were included if they could be used to inform the Commissioning Policy on BAHAs.

Results: All studies referenced by the policy were graded as 'low quality' or 'very low quality' evidence. The strength of the overall policy was graded as weak. The literature cited by the Commissioning Policy contained several areas of disagreement with the Commissioning Policy itself.

Nineteen articles were included following systematic review. These studies identified six areas for development of the NHS Commissioning Policy for BAHAs: 1) BAHA implantation in children with unilateral hearing loss; 2) BAHA as an alternative to other surgical treatments; 3 ) The minimum number of BAHAs implanted by a centre each year; 4) Unilateral BAHA implantation in patients with less than profound sensorineural hearing loss; 5) Bilateral BAHA implantation in adults; 6) BAHA implantation in patients with osteogenesis imperfecta.

Conclusion: It is important that these areas are reviewed by the commissioning board to help ensure equitable access to BAHA services and that resources are allocated effectively. It is also clear that high quality research is urgently needed in this field to help inform national policy.

doi:10.1017/S0022215116006204

\section{ID: IP124}

\section{Couplers for Vibrant Soundbridge ${ }^{\circledR}$ \\ implant vs no-Coupler-Vibrant \\ Soundbridge ${ }^{\circledR}$ implant}

Presenting Author: Manuel Manrique

Manuel Manrique

$N A$

Introduction: The middle ear active implant Vibrant Soundbridge ${ }^{\circledR}$ (VSB) is a device designed for the treatment of the sensorineural, mixed and conductive hearing losses. Depending on the type of the hearing loss and the anatomical condition of the different middle ear structures, the placement of its FMT can be carried out in different ossicular chain points or directly on the round or oval window, aimed to obtain a direct stimulation of the inner ear. Recently, new Couplers have been designed to obtain a better coupling of the FMT with these structures.

Objectives: To compare surgical feasibility and auditory performance with VSB traditional system versus the new "Couplers" for the VSB implant

Methods and materials: Thirty eight patients treated with VSB systems are included at the moment. Eleven patients implanted with VSB Coupler versus 27 patients with no-Coupler VSB. Three out of eleven VSB Coupler implants were indicated for sensorineural hearing loss (SNHL) patients and eight of them for conductive and mixed hearing loss patients. Regarding no-Coupler VSB, seven patients were diagnosed of SNHL whereas twenty of conductive and mixed hearing loss patient. 